\title{
From e-learning to s-learning: A Review
}

\author{
Anam Saiyeda \\ Department of Computer Science and Engineering \\ Jamia Hamdard, New Delhi-62, India \\ anamsaiyeda@jamiahamdard.ac.in
}

\begin{abstract}
With technology changing at fast rates, learning has transformed a lot. Technology has been incorporated into education, to enable better learning and teaching. Every kind of information is now available just a click away. Education is not limited to just simple YouTube lectures. Interactive apps, quizzes, discussions, software have now enhanced learning and expanded the field to include topics like e-learning, s-learning, $u-$ learning, m-learning. This work reviews the work done in these fields to highlight how education sector has evolved from basic e-learning to smart campuses and s-learning. This will enable educators, policy makers, students and researchers to gain insight into the latest trends in this sector. The paper also categorizes the various techniques to create some categories and find out the advantages and research gaps in this field.
\end{abstract}

Keywords: e-learning, m-learning, s-learning, i-learning, learning analytics, educational data mining, data mining.

\section{Introduction}

The world is moving towards making everything 'smart', be it phones, devices, vehicles, cities, campuses. Smart campus is a subset of the smart cities initiative attempting to equip universities, colleges and educational institutes with ubiquitous and smart technology. The Cambridge dictionary defines a smart machine as one that uses computers to make it work, in order to make it act in an independent way. The approaches used for smart campus are categorized as technology driven, organization development based and smart city concept adoption [1]

These smart campuses generate large amounts of data in the form of sensor data, activity, software etc. This can be utilized to gain insights into the working of campuses and find patterns. In the field of education particularly this data can be extremely valuable. Starting from e-learning we moved on to m-learning and now s-learning an i-learning.

Now we are moving towards intelligent learning in the smart campuses. Just the simple incorporation of technology on the campus does not make it smart or intelligent. The technology has to actually aid in making things better. The intelligent campus upgrades the smart era to an intelligent era. The i-campus is not just smart but also intelligent, which has the faculty of thinking, reasoning and understanding, with the capability of not only making adjustments but also learning and adapting in response to the changing circumstances.

The concept of i-campus was initially introduced by MIT in the MIT-Microsoft Alliance program. Its aim was to revolutionize the practice of higher education [2]. 
In the education sector learning analytics and educational data mining are two fields which have contributed a lot towards improved learning. Learning analytics aims to improve and optimize learning by collection and analysis of data about learners, learning experiences, and learning programs. LA aims at understanding the whole system and focuses on leveraging human judgment. It informs and empowers students and instructors. It focuses on analysis, sentiment analysis, influence analysis, discourse analysis, learner success prediction, concept analysis and sense-making models [3].

Educational Data Mining (EDM)[4] is an emerging interdisciplinary research area that deals with the usage of methods to explore data originating in an educational context. It studies educational data generated by students and instructors using statistical, machine-learning and data-mining methods. It helps in optimization of learning processes.

This paper presents a thorough review of the work done in the field of s-learning and ilearning in smart campus. The introduction is followed by the section on research method. The third section presents a thorough literature review followed by results and conclusion.

\section{Research Method}

\subsection{Need for review}

Exhaustive reviews are requires for recognizing shortcomings and gaps in existing methods. This section presents the work done in this field previously. Classification of the studies based on their titles is the initial purpose. It takes into consideration many primary and secondary studies in this field.

\subsection{Sources of information}

In order to carry out the review quality databases were searched. The short listing was done as per the guidelines settle upon in advance, concerning the aim of this paper. The papers were selected from the databases of ACM, IEEE, Springer and Elsevier.

\subsection{Literature search strategy}

The keywords <smart education>, <intelligent learning> and <educational data mining> were used. The search was further refined with the keywords <Computer Science $>$ and <learning analytics $>$. Irrelevant papers from other fields and disciplines were removed and the results summarized. Only papers from the last 5 years were considered i.e. from the year 2014 to 2018.

The papers were ordered on the basis of relevance. After refining the search for the specified duration the titles were considered for narrowing down the papers. Then abstracts were studied to further filter out papers and select relevant ones. 


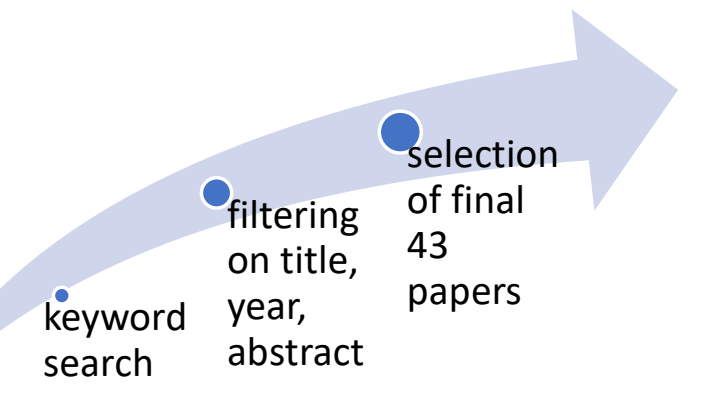

Fig 1: Selection of papers from the databases

\subsection{Study selection}

The year of paper publication was the first criteria used to reduce the sample set. Then based on keywords papers were selected. The titles were used to further narrow down the selection. Then the abstracts of the papers were analyzed to choose the final set of papers.

\subsection{Data extraction}

Kitchenham[5] guidelines were consulted for data gathering and extraction. The quality of the papers was the prime benchmark used for final paper selection. For the study 43 papers were selected.

\subsection{Research questions}

The purpose of this review is to study the various methods and techniques employed for improving education in any university, college or educational institute using technology. To summarize the various ways in which education is being improved utilizing machine learning, IoT, s-learning and so on. The study aims to summarize the advantages, shortcomings and research scope of these techniques to help the researchers in this field. The following research questions were formulated

RQ1. What are the various ways being employed in computer science and engineering to improve education?

RQ2. What are the advantages offered by these methods?

RQ3. What are the research gaps in this field?

\section{Literature Review}

This literature review includes the summary of 43 papers and focuses on their techniques used and shortcomings or research gaps. The papers utilized a wide array of methods and thus have been grouped according to some criteria. The criteria for grouping are based on the technique applied by the authors in their papers previously. Based on techniques we have improving 
education by the use of LMS, using Machine learning techniques, using game based methods, using mobile-learning, using activity tracking and IoT devices, and using social media.

\subsection{LMS and e learning}

To aid the process of learning and teaching software aids have been widely used. e-Learning has existed for a long time and is a basic ways to enhance education by using technology. LMS or learning management systems is the umbrella term covering all these techniques. Elearning involves not just the use of LMS but also various forms of online learning mechanisms. The instructor and learner can interact through the use of Information and Communication Technologies(ICT). Learning management systems (LMS) are software applications used for the administration, documentation, tracking, reporting and delivery of educational courses, training programs, or learning and development programs. Moodle [6], Blackboard [7] and Shakai [8] are some of the most widely used LMS. Several research papers have utilized the data generated from these LMS and used it by applying data mining and machine learning techniques to obtain patterns and make predictions regarding the performance of students etc.

A paper used the logs of the LMS like BlackBoard for finding the rate of student involvement in the learning module. Association, clustering and prection were the tecniques used. [9] Seventeen blended courses were anlaysed with 4,989 students in an institution using Moodle LMS. The student performance was predicted from LMS using its logs. Regression was used for this[10]. JRIP , J48 and Bayesian Network classification techniques used to predict the student's performance by analyzing their behavior from the Moodle LMS.[11] Tracked the progress of students and predicted Students' Weekly Achievement During a Circuits and Electronics MOOC. Regression was applied for this [12].

With the wide availability of internet and most people being online all the time be it students or educators social media, YouTube, and other online activities provide a very good method to study the behavior of students. Insights can be obtained about how students behave online, access course materials, discuss educational topics online or on social media. Applications have been developed which utilize this activity information to build recommendation systems, reminders, motivation to students to spend more time studying.

\subsection{M learning}

M-learning, is an educational system which stands for mobile learning. With the advent of smart phones and internet everyone has access to android and i-OS based phones. Using these mobile devices, it's very easy to have a continuous access to the learning resources in the form of apps, e-books, YouTube and other such sources. But studies show that tracking activities through smart phones is limited. Earlier work has been done using SMS to improve learning [12]. But the research in these is still emerging. This field has a lot of potential. Mobile Learning (m-learning) technique which utilised Short Message Service (SMS) to increase student participation in the course. It showed that students who received mobile messaging showed better performance than the other students. Records of Moodle provided the data [13] Social network analysis techniques were used to provide a framework to support educational 
decision making in mobile learning. A task-interaction framework was provided supporting educational decision-making in mobile learning.[14]

The technology and demand feasibility of mobile learning platform was analysed in this paper. An Android based hybrid architecture was proposed for intelligent mobile learning platform. Also the prototype of mobile learning front-end platform was given[15]. This paper uses mobile applications eg rain classrooms and UMUs, to develop intelligent teaching content, build an intelligent teaching platform, and building a flipped classroom-style intelligent teaching model [16]. The paper studied the technology and demand feasibility of mobile learning platform. It designed an android based hybrid architecture mode for intelligent mobile learning platform. A prototype of mobile learning front-end platform was also designed. [17]

\subsection{Game based}

Student Proficiency Inferred from Game data (SPRING) modeled game playing behavior in educational games. Logs of students playing educational mini-games provided the data on which Hidden Markov Models applied. regression model applied to it to predict the score[18]. This paper created a game based on an interactive storybook encouraging learners to create their own unique stories and thus improve learning.[19]

\subsection{ML methods}

Multi layer perceptron was used to predict a student's performance [20]. Student's personal information like family expenditures, family background etc are used for applying Learning analytics, discriminative and generative classification models. SVM, Regression Tree, Naïve Bayes Classifier are the techniques applied. These are used to predict whether a student will be able to complete his degree or not[21]. This paper used online learning material to predict academic success of student. Machine learning methods applied to find relation between extent of material usage and course outcomes. The techniques of support vector classifiers and support vector regressors were sued for this. The time spent with each paragraph of the online learning material was recorded to make predictions[22]

Another work utilized SVM(Linear), SVM(RBF),Logistic Regression to perform classification. facial expressions, head pose and eye gaze were used to gather the the engagement or attention level of the students. [23] A paper used clustering for under-graduate students data. students were grouped according to their final marks they achieved in the course. [24] Another applied k means clustering to text documents, images and videos to support e-learning.

The input for clustering algorithm was the frequency of the common unique words. It was used to predict student behavior. using student motivated engagement profiles [25] . Used multi-regression models personalized to each student. Features related to student's past performance were considered incluidng engagement and course characteristics. [26]

An Architecture called WAVE was proposed for Predicting Dropout in Undergraduate Courses using EDM techniques to predict and to identify students at dropout risk. student identification (id), course id, year and semester of admission,CGPA, number of credits were some of the attributes considered. Naïve Bayes (NB), Multilayer Perceptron (MLP),Support Vector Machine and RBF kernel (SVM2) and Decision Table (DT) were the methods used 
[27]. Another paper presents an innovative approach to promoting AI in Education. Smart sensors and wearable devices use for self-regulated learning and use of learning analytics and machine learning algorithms[28].

The paper proposed a learner's emotion recognition model. This worked in the online learning scenario and built an intelligent education system called Smart-E. Deep neural network and support vector machine have been used[29]

This paper designed a student achievement predicting framework. It included data processing and student achievement predicting. A layer-supervised multi-layer perceptron (MLP)-based method was proposed[30]

Another work used the techniques of NLP[31], Built recommender system[32], Robot Based On Bot Framework[33], proposed skill-based e-learning, the fuzzy knowledge model[34]

\subsection{Other methods}

Social media and tracking the usage of learning apps can help educators improve teaching and learning and make the process more interactive. Chen et al[35] used Facebook to explore differences in student learning outcomes and satisfaction depending on various learning styles. This is an emerging field with many ways available which can be tapped to optimize learning.

Other methods include a variety of techniques like tracking game logs of students and using it to predict their performance. Preparing questionnaires for students, tracking them by regular feedback, and using other techniques like tracking physical fitness to study the relation between physical health and student performance [36]. Another can be finding the links between stress, mental health [37] and education and so on.

The logs of LMSs particularly Moodle are widely used to track the activities and perform classification, clustering, and prediction on the data generated. BlackBoard is another LMS used. Some techniques use mobile phones to try and optimize the learning experience. Game Activity Logs are also used to predict student performance and make predictions. Regression, multi-layer perceptron(MLP),Support Vector Machine (SVM) [discriminative], Naive Bayes (NB), K-means clustering, Bayesian Network, neural network classification model are some of the techniques employed. The techniques are broadly classified into the ones using softwares like LMS to track activities, tracking activities online which also includes social media, use of mobiles and apps for such activities, and other methods like game logs, questionnaires etc.

Smart Hybrid Learning Method produced a model of Smart Hybrid Learning Method. It is the evolution of the flipped classroom method whihc has been combined with several other learning method approaches, such as Challenge Based Learning and Case Based Learning. It allows learning inside as well as outside the classroom. [38.]

Another paper did a study on blockchain driven smart education .Its related system frameworks and key technologies were analyzed[39.]use of IoT in education. objects with sensors, wearable technologies etc collect data with and intra-communication is taking place through cloud computing. [40] Did a study on the smart education environment. [41] A thorough study of smart learning environments[42.]An important part of smart campus is 
campus activities, and teaching performance evaluation scientifically which is done here[43] Papers [44],[45][46] did a thorough review of LA

\section{Results}

43 papers were studied for this review. The results categorize them on the basis of techniques applied. 5 groups have been created according to the methodologies mainly LMS based, game based, m-learning, utilizing machine learning and others category. LMS based includes the techniques to improve education and learning by the use of Learning management systems or LMS and enhancing the e-learning techniques. Game based include various educational games to optimize learning and teaching. Mobile based or m-learning includes the mobile based techniques utlising apps, SMS, and activities or logs of mobiles. Machine learning includes the various techniques of ML applied to improve learning and has the maximum amount of work done. Others includes the various other techniques like social media etc to improve education. The figure below gives a pictorial representation of these techniques.

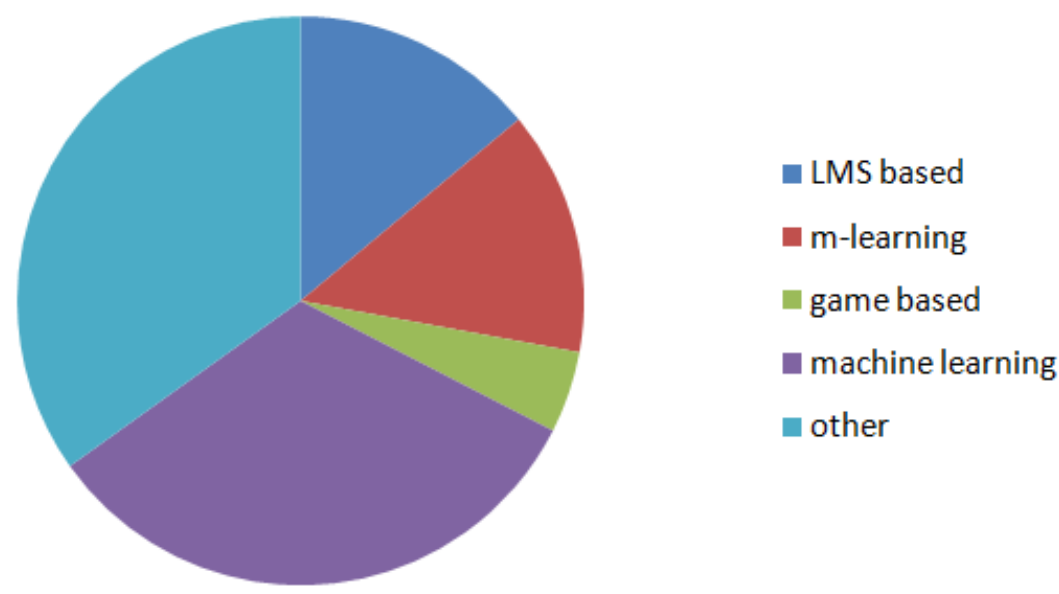

Fig 2. The techniques applied categorized

The duration of 5 years i.e. from 2014 to 2018. The number of papers selected from each year have been presented below. In 2018 highest number of papers were published in this field, followed by In the year 2016 . 


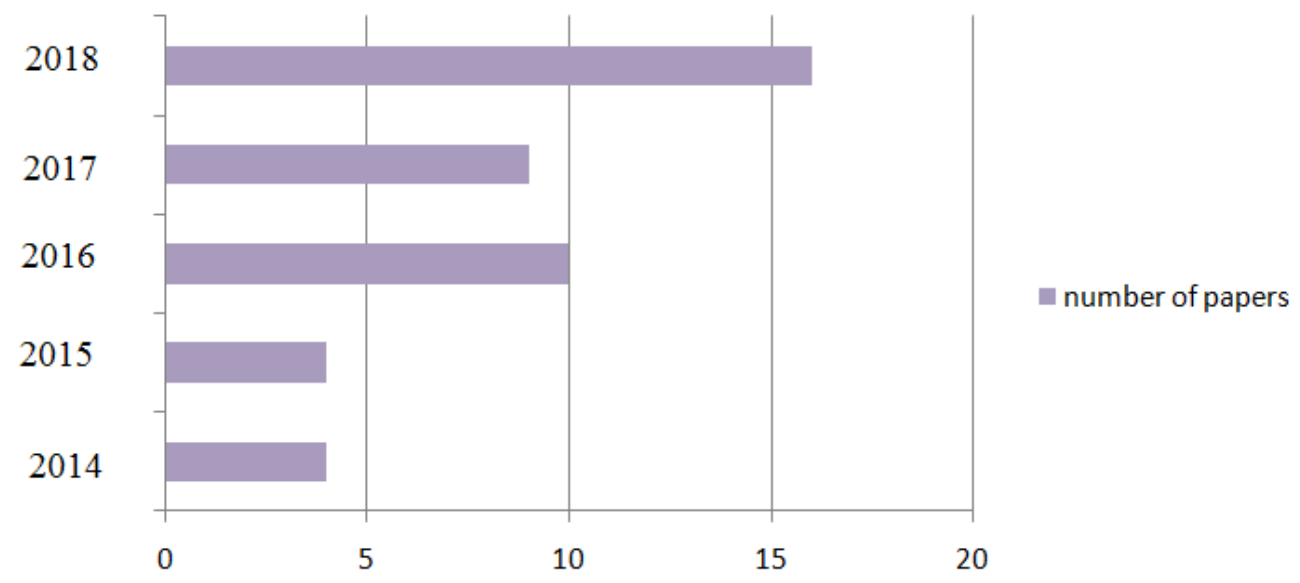

Fig 3. The number of papers selected for review per year

The results show that the maximum work has been done in the field of machine learning. Mobile based or m-learning is an upcoming and very promising field in which work can be done. It includes both game based and other m-learning methods. This is because mobiles have become ubiquituius and are widely used by all so it's a very promising field with a lot of potential.

\section{Conclusion}

This paper has provided an insight into the current trends of this fields. This review which is based on some longer reviews, has summarized the literature in this field. The analysis has shown that most of the work has been done in this field using ML techniques. The field of mobile learning is an emerging field in which more work can be done. This is the research gap identified. Mobiles are the most used technological devices; hence they are a mine of huge datasets. They can provide lots of data in the form of sensor, GPS, activity data of app usage and so on. All this can be helpful in optimizing and improving the education system. Improved education will lead to better ranking of institutes, development of smarter teaching and learning methods, a smarter an intelligent campus and thus smarter cities. 


\section{References}

[1] Muhamad, Wardani, Novianto Budi Kurniawan, and Setiadi Yazid. "Smart campus features, technologies, and applications: A systematic literature review." 2017 International Conference on Information Technology Systems and Innovation (ICITSI). IEEE, 2017.

[2] The iCampus project under MIT-Microsoft Alliance: http://icampus.mit.edu

[3] Siemens, George, and Ryan SJ d Baker. "Learning analytics and educational data mining: towards communication and collaboration." Proceedings of the 2nd international conference on learning analytics and knowledge. ACM, 2012.

[4] Romero, Cristóbal, and Sebastián Ventura. "Educational data mining: a review of the state of the art." IEEE Transactions on Systems, Man, and Cybernetics, Part C (Applications and Reviews) 40.6 (2010): 601-618.

[5] Kitchenham, Barbara. "Procedures for performing systematic reviews." Keele, UK, Keele University 33.2004 (2004): 1-26.

[6] Rice, W. H. "9. E-Learning Course Development: A complete guide to successful learning using Moodle 1.9." (2008).

[7] Boeren, Ellen. "The Blended Learning Environment in Higher Education: The Tutor and Student Perspective." Handbook of Research on Learning Outcomes and Opportunities in the Digital Age. IGI Global, 2016. 180-197.

[8] Berg, Alan Mark, and Michael Korcuska. Sakai courseware management: The official guide. Packt Publishing Ltd, 2009

[9] Hussain, Mohammed, et al. "Mining educational data for academic accreditation: aligning assessment with outcomes." Global Journal of Flexible Systems Management 18.1 (2017): 51-60

[10] Hussain, Mohammed, et al. "Mining educational data for academic accreditation: aligning assessment with outcomes." Global Journal of Flexible Systems Management 18.1 (2017): 51-60

[11] Sanchez-Santillan, Miguel, et al. "Predicting students' performance: Incremental interaction classifiers." Proceedings of the Third (2016) ACM Conference on Learning@ Scale. ACM, 2016.

[12]DeBoer, Jennifer, and Lori Breslow. "Tracking progress: predictors of students' weekly achievement during a circuits and electronics MOOC." Proceedings of the first ACM conference on Learning@ scale conference. ACM, 2014.

[13] Marçal, Edgar, et al. "Mobile phone text messaging to increase student participation: an experience in a blended course." Proceedings of the 31st Annual ACM Symposium on Applied Computing. ACM, 2016

[14] Arrigo, Marco, Giovanni Fulantelli, and Davide Taibi. "Challenges of Using Learning Analytics Techniques to Support Mobile Learning." International Association for Development of the Information Society (2015).

[15]Qiu, Deming. "Development and Implementation of Learning System of an Intelligent Learning System for Ideological and Political Education in Colleges under Mobile Platform." 2018 International Conference on Virtual Reality and Intelligent Systems (ICVRIS). IEEE, 2018.

[16]Lu, Fang, Zhen Liu, and Zejiang Liu. "Smart Phone Endowed Intelligent Teaching for University General Education Curriculum in China." 2018 IEEE International 
Conference on Teaching, Assessment, and Learning for Engineering (TALE). IEEE, 2018

[17]Qiu, Deming. "Development and Implementation of Learning System of an Intelligent Learning System for Ideological and Political Education in Colleges under Mobile Platform." 2018 International Conference on Virtual Reality and Intelligent Systems (ICVRIS). IEEE, 2018.

[18]Falakmasir, Mohammad H., et al. "A data-driven approach for inferring student proficiency from game activity logs." Proceedings of the Third (2016) ACM Conference on Learning@ Scale. ACM, 2016

[19] Smart, Kathleen. "SIGCHI Extended Abstract: The Frog's Princess Interactive Storybook." Proceedings of the 2018 Annual Symposium on Computer-Human Interaction in Play Companion Extended Abstracts. ACM, 2018.

[20] Qu, Shaojie, Kan Li, Shuhui Zhang, and Yongchao Wang. "Predicting Achievement of Students in Smart Campus." IEEE Access 6 (2018): 60264-60273

[21] Daud, Ali, et al. "Predicting student performance using advanced learning analytics." Proceedings of the 26th International Conference on World Wide Web Companion. International World Wide Web Conferences Steering Committee, 2017

[22]Leppänen, Leo, et al. "Predicting Academic Success Based on Learning Material Usage." Proceedings of the 18th Annual Conference on Information Technology Education. ACM, 2017

[23]Leppänen, Leo, et al. "Predicting Academic Success Based on Learning Material Usage." Proceedings of the 18th Annual Conference on Information Technology Education. ACM, 2017

[24] Mishra, Akansha, Rashi Bansal, and Shailendra Narayan Singh. "Educational data mining and learning analysis." Cloud Computing, Data Science \& EngineeringConfluence, 2017 7th International Conference on. IEEE, 2017

[25]Eck, Adam, Leen-Kiat Soh, and Duane F. Shell. "Investigating differences in wikibased collaborative activities between student engagement profiles in CS1." Proceedings of the 47th ACM Technical Symposium on Computing Science Education. ACM, 2016.

[26] Elbadrawy, Asmaa, R. Scott Studham, and George Karypis. "Collaborative multiregression models for predicting students' performance in course activities." Proceedings of the Fifth International Conference on Learning Analytics And Knowledge. ACM, 2015

[27] Manhães, Laci Mary Barbosa, Sérgio Manuel Serra da Cruz, and Geraldo Zimbrão. "WAVE: an architecture for predicting dropout in undergraduate courses using EDM." Proceedings of the 29th Annual ACM Symposium on Applied Computing. ACM, 2014

[28] Ciolacu, Monica, et al. "Education 4.0-Artificial Intelligence Assisted Higher Education: Early recognition System with Machine Learning to support Students' Success." 2018 IEEE 24th International Symposium for Design and Technology in Electronic Packaging(SIITME). IEEE, 2018.

[29] Li, Gang, and Yaoying Wang. "Research on leamer's emotion recognition for intelligent education system." 2018 IEEE 3rd Advanced Information Technology, Electronic and Automation Control Conference (IAEAC). IEEE, 2018.

[30]Qu, Shaojie, et al. "Predicting Achievement of Students in Smart Campus." IEEE Access 6 (2018): 60264-60273. 
[31]Lee, Sarah Priscilla, et al. "Utilizing Natural Language Processing (NLP) to Evaluate Engagement in Project-Based Learning." 2018 IEEE International Conference on Teaching, Assessment, and Learning for Engineering (TALE). IEEE, 2018

[32] Hassan, Mohammed, and Mohamed Hamada. "Smart media-based context-aware recommender systems for learning: A conceptual framework." 2017 16th International Conference on Information Technology Based Higher Education and Training (ITHET). IEEE, 2017.

[33] Qi, Chuanwei. "The Application Study On The Customer Service Chatting Robot Based On Bot Framework." 2017 2nd Joint International Information Technology, Mechanical and Electronic Engineering Conference (JIMEC 2017). Atlantis Press, 2017.

[34] Gomathi, C., and V. Rajamani. "Skill-based education through fuzzy knowledge modeling for e-learning." Computer Applications in Engineering Education 26.2 (2018): 393-404.

[35] Chen, Yu-ching. "Linking learning styles and learning on mobile Facebook." The International Review of Research in Open and Distributed Learning 16.2 (2015).

[36] Hills, Andrew P., Donald R. Dengel, and David R. Lubans. "Supporting public health priorities: recommendations for physical education and physical activity promotion in schools." Progress in cardiovascular diseases 57.4 (2015): 368-374.

[37] Wang, Rui, et al. "StudentLife: assessing mental health, academic performance and behavioral trends of college students using smartphones." Proceedings of the 2014 ACM international joint conference on pervasive and ubiquitous computing. ACM, 2014.

[38] Hartono, Sugiarto, et al. "Smart Hybrid Learning Framework Based on Three-Layer Architecture to Bolster Up Education 4.0." 2018 International Conference on ICT for Smart Society (ICISS). IEEE, 2018

[39] Gong, Xiaoyan, et al. "Parallel-Education-Blockchain Driven Smart Education: Challenges and Issues." 2018 Chinese Automation Congress (CAC). IEEE, 2018

[40] Majeed, Asim, and Mahmood Ali. "How Internet-of-Things (IoT) making the university campuses smart? QA higher education (QAHE) perspective." 2018 IEEE 8th Annual Computing and Communication Workshop and Conference (CCWC). IEEE, 2018.

[41] Abdel-Basset, Mohamed, et al. "Internet of things in smart education environment: Supportive framework in the decision-making process." Concurrency and Computation: Practice and Experience (2018): e4515

[42] Dron, Jon. "Smart learning environments, and not so smart learning environments: a systems view." Smart Learning Environments 5.1 (2018): 25

[43] Xu, Xin, Yunsheng Wang, and Shujiang Yu. "Teaching Performance Evaluation in Smart Campus." IEEE Access 6 (2018): 77754-77766

[44] Viberg, Olga, et al. "The current landscape of learning analytics in higher education." Computers in Human Behavior (2018)

[45]Zhuang, Rongxia, et al. "Smart learning environments for a smart city: from the perspective of lifelong and lifewide learning." Smart Learning Environments 4.1 (2017):

[46] Masran, Saiful Hadi, et al. "Effectiveness of using an interactive media in teaching and learning: A case study." 2017 IEEE 9th International Conference on Engineering Education (ICEED). IEEE, 2017 